\title{
Neuropsychiatric symptoms in elderly - a short practical approach
}

\begin{tabular}{c} 
Irene Davidescu \\
2 $\begin{array}{c}\text { nd Department of Neurology, Colentina Clinical Hospital, } \\
\text { "Carol Davila" University of Medicine and Pharmacy, Bucharest, Romania }\end{array}$ \\
\hline
\end{tabular}

\begin{tabular}{|l} 
ABSTRACT \\
Life expectancy increases neurological and psychiatric pathology in the elderly. Neurocognitive disorders have an \\
increasing incidence and therefore Alzheimer's disease and vascular dementia become a common pathology. \\
Delirium is a quite frequent symptom in general practice and managing agitation in elders can become challenging \\
for the doctor, especially because of presence of comorbidity and sometimes of paradoxical reactions due to cerebral \\
vulnerability. \\
Neuropsychiatric symptoms are a challenge in management of patients with neurodegenerative disorders, being a \\
real burden for patients themselves, caregivers and medical staff. \\
A concise guide for managing these symptoms with non-pharmacological methods and drugs allowed in such condi- \\
tions. \\
$\quad$ Keywords: neuropsychiatric symptoms, behavioral, non-pharmacological management,
\end{tabular}
allowed medication

\section{INTRODUCTION}

The spectrum of neuropsychiatric symptoms is very wide, with positive and negative symptoms, and incidence correlates with severity of the disease, almost $95 \%$ of patients with dementia developing behavioral and psychological symptoms during the course of the disease $(1,2)$, such as:

- aggression;

- agitation;

- anxiety;

- apathy;

- appetite/eating changes;

- delusions;

- depression;

- disinhibition;

- euphoria;

- hallucinations;

- irritability/emotional lability;

- motor disturbances and stereotyped behaviors;

- night-time behaviors/sleep disturbances.
Some symptoms that patients might have can get very unpleasant for caregivers (3), such as:

- irritability;

- agitation, with disruptive behaviors and aggression/screaming;

- sleep disturbances;

- anxiety;

- apathy;

- delirium;

- delusions.

There are a lot of factors that can affect appearance of these symptoms, as:

- clinical conditions: acute infections, dehydration, pain, hypoxia, constipation;

- drug induced: anticholinergics, benzodiazepines, tricyclic antidepressants;

- psychological: loneliness, frustration, inability to communicate, unfamiliarity with setting/people, changes of the environment (hospital admissions); 
- environmental: positional discomfort, disrupted routines, inappropriated light, sensory deficits, noise.

There is no need to aggressive treat all behaviors symptoms, as they are considered benign:

- when there is no harm for the patients or others;

- if the behavior is manageable and occurs for short time and infrequent;

- if behavior stops easily with an appropriate intervention;

- if it doesn't cause distress to the patient or caregivers.

Assessment implies checking conditions of appearance:

- Medical conditions: everything with an acute onset, may indicate a new medical condition which can destabilize finally patient's mental status;

- Unmet needs: hunger, thirst, pain, immobility;

- Environmental problems: light levels, roommate, over or under-stimulation.

\section{NON-PHARMACOLOGICAL APPROACHES}

Management of neuropsychiatric symptoms has several factors that are potentially modifiable (4):

- in the patient: seeking for managing all acute intercurrent medical illness, unmet needs (pain, sleep disturbances, fear, boredom) or sensory deficits;

- in the caregivers: stress, depression, emotional upset, little knowledge about the disease, prejudices and lack of social support;

- in the environment: clutter, over or under-stimulation, lack of daily routine, activity of daily life.

DICE (Describe, Investigate, Create and Evaluate) approach is a step by step plan to manage these patients, involving non-pharmacological and drug interventions (5). Anamnesis is very important, taken both from the patient and caregivers and doctor has to be patient and ask all questions needed so that finally a management plan to be implemented. Any escalation of aggression must be closely monitored, to ensure its suspension. Steps of escalation of behavioral symptoms are:

- emotional tension and restlessness in the environment,
- non-verbal signs as facial expression of contempt and disdain;

- verbal aggression in form threats of violence

- violence against objects;

- violence against persons.

To ensure de-escalation in case of tension and anxiety in such mentally disordered patients we must:

- keep physical distance;

- keep conversation going with neutral tone and allow decrease tension;

- lead patient out of the field of tension;

- act unexpectedly like using humor;

- do not debate but suggest alternatives.

There are some non-pharmacological measures that can be really effective, such as:

- environmental adjustment;

- caregiver education;

- cognitive training;

- sensory: light therapy, massage, evening spa, music therapy.

\section{MEDICATION}

Atipical antipsychotics: adverse events offset advantages in efficacy (6); they induce a 2-3 times increased cerebrovascular adverse events risk and $1-2 \%$ increased risk death (7); risperidone is the only licensed drug for treatment of aggression and delusions, but other drugs can be also used, even though adverse events may offset advantages in efficacy (6).

\begin{tabular}{|c|c|c|}
\hline drug & start & range \\
\hline RISPERIDONE & $0.25 \mathrm{mg}$ & $0.5-2 \mathrm{mg} /$ day \\
\hline OLANZAPINE & $2.50 \mathrm{mg}$ & $2.5-10 \mathrm{mg} /$ day \\
\hline QUETIAPINE & $25 \mathrm{mg}$ & $25-100 \mathrm{mg}$ \\
\hline ARIPIPRAZOLE & $2 \mathrm{mg}$ & $5-10 \mathrm{mg}$ \\
\hline
\end{tabular}

Whenever needed to be prescribed we must (8):

- consider first non-pharmacological methods;

- assess all cardiovascular associated risk factors;

- evaluate risks and benefits for patients and caregivers;

- identify target symptoms as psychosis, hostility, aggression.

Antidepressants: there is modest evidence efficacy in dementia, the only symptom that can be favorable address is agitation, and trazodone (seroto- 
nin receptor antagonists and reuptake inhibitors -SARIs) is the first choice (50-150 mg), even thought other selective serotonin reuptake inhibitors (SSRIs) may tapper agitation (citalopram N.B. not available in our country).

Anticonvulsivants: carbamazepine and valproate can be used for a second line or in association with an atypical antipsychotic (9).

\begin{tabular}{|c|c|r|}
\hline drug & & \\
\hline CARBAMAZEPINE & $100 \mathrm{mg}$ & $2-4$ mes a day \\
\hline VALPROAT & $150-300 \mathrm{mg}$ & $2-3$ mes a day \\
\hline
\end{tabular}

Benzodiazepines: they reduce agitation but there are adverse effects as: falls, sedation or worsening cognition; it is better not to use them, or at least to try to use them as little as possible (10).

\begin{tabular}{|c|c|c|}
\hline drug & start & max \\
\hline LORAZEPAM & $0.5-1 \mathrm{mg}$ & $4-6$ mes a day \\
\hline OXAZEPAM & $7.5-15 \mathrm{mg}$ & 4 mes a day \\
\hline
\end{tabular}

We must not forget medication side effects (especially benzodiazepines) which can lead to:

- recent falls;

- increase in confusion;

- increased anxiety and agitation;

- excessive sleep/seems sedated or decreased sleep;

- increased unsteadiness on their feet;

- any change in their level of function.

In the same time we must be attentive to (11):

- consistent dosage of drugs through changes in body mass;

\section{REFERENCES}

1. Cerejeira J., Lagarto L., Mukaetova-ladinska EB: behavioral and Psychological Symptoms of Dementia. Front Neurol 2012; 3:73

2. Wint D., Cummings J.L. Neuropsychiatric aspects of cognitive impairment. In: Husain M, Schott J, eds. Oxford Textbook of Cognitive neurology and Dementia. Oxford: Oxford University Press, 2016:197-208

3. Rocca P., leotta D., Liffredo C. et al. Neuropsychiatric symptoms underlying caregiver stress and insight in Alzheimer's disease. Dement Geriatr Cogn Disord 2010; 30:57-63

4. Kales H.C., Gitlin L.N., Lyketsos C.G. Assessment and management of behavioral and psychological symptoms of dementia. BMJ 2015; 350:h369-h369

5. Kales H.C., Gitlin L.N., Lyketsos C.G. Detroit Expert Panel on Assessment and Management of Neuropsychiatric Symptoms of Dementia. Management of neuropsychiatric symptoms of dementia
- recent increased dose or changes in timing of dose administration;

- too many associated drugs;

- low compliance or supra-dosing;

- any new medication recent established for dementia or other pathologies, causing drug interactions;

- over the counter meds which can interfere with medication;

- alcohol withdrawal.

\section{CONCLUSIONS}

- Any psychological changes in the behavior of a dementia patient has to be closely monitored and checked for any external factors that can lead to escalation, trying to correct reversible factors.

- Non-pharmacological measures must be of first choice, trying to de-escalation of behavioral symptoms.

- Atypical antipsychotics may be used, but started with low doses and titrated up to the minimum effective dose tolerated and if there is a positive response, tapering the doses and withdrawn must be done during 4 months and afterwards assessment of behavioral changes must be done at least another 4 months for identifying signs of recurrence (12).

- Benzodiazepines can be effective but it is preferable not to use them as long as side effects can be augmented by cerebral vulnerability in these patients.

Conflict of interest: none declared Financial support: none declared

in clinical settings:recommendation from a multidisciplinary expert panel. J Am Geriatr Soc 2014; 62:762-9

6. Clinical Antipsychotic Trial of Intervention Effectiveness-Alzheimer's Disease. Rosenheck, Cost bnefit analysis; Arch Gen Psychiatry 2007; 64(11):1259-1268

7. Yager J., Kunkle R., Fochtmann L.J. et al. Who's your expert?Use of an expert opinion survey to inform development of American Psychiatric Association practice guidelines. Acad Psychiatry 2014; 38(3):376-382

8. Marder S.R. 2006. A review of agitation in mental illness:Treatment guidelines and current therapies. J Clin Psychiatry. 67 (Suppl 1):13-21.

9. Asadollahi S., Heidari K., Hatamabadi H. et al. 2015. Efficacy and safety of valproic acid versus haloperidol in patients with acute agitation. Int Clin Psychopharmacol. 30:142-150. 
10. Wilhelm S., Schacht A., Wagner T. 2008. Use of antipsychotics and benzodiazepines in patients with psychiatric emergencies: results of an observational trial. BMC Psychiatr. 8:61

11. Ford A.H., Almeida O.P. 2015. Pharmacological interventions for preventing delirium in the elderly. Maturitas. 81:287-292. Available from:http://www.ncbi.nlm.nih.gov/pubmed/ 25890587
12. The AMERICAN PSYCHIATRIC ASSOCIATION Practice Guideline on the use of Antipsychotics to Treat Agitation or Psychosis in Patients with Dementia; appi.books.9780890426807 